\title{
SEROPREVALENCE AND ASSOCIATED FACTORS OF HELICOBACTER PYLORI AMONG MYANMAR CHILDREN FROM THE PERI-URBAN COMMUNITY OF YANGON
}

\author{
Phyu Phyu Win \\ Paediatric Department, Faculty of Medicine and Health Sciences, UCSI University, Malaysia \\ Corresponding author: Phyu Phyu Win \\ Email: drppwin@gmail.com
}

\begin{abstract}
Helicobacter pylori infects $50 \%$ of children aged 5 years in developing countries. The infection is associated with socioeconomic background and family composition. The aim of this study was to determine the seroprevalence and associated factors of Helicobacter pylori infection among Myanmar children in Insein Township, which is a peri-urban community situated in the northern region of Yangon. A cross-sectional study was carried out in Insein Township on 193 children, aged 2 to 13 years, who were tested for $H$. pylori antibody by enzyme-linked immunosorbent assay (ELISA) test. Parents were interviewed using a questionnaire. Statistical analyses were done using chi-square test and twosample t-test. The prevalence of $H$. pylori infections was $67.36 \%$. The mean age of the participants was $7.6+/-3.85$ years. Those positive for $H$. pylori antibody were significantly older $(8.18+/-3.8)$ than those with negative results (6.41+/-3.7). There is no gender preponderance. The sero-positive rate was higher among low socio-economic group. The children who tested positive for $\mathrm{H}$. pylori antibody had a mean family member of 5.97+/-2.22 and those who tested negative had 6.06+/-2.17. Thus, there was no association between $H$. pylori infection and number of family members. There was no association between $\mathrm{H}$. pylori infection and overcrowding, type of drinking water, type of latrine used and type of housing. Our findings suggest that the prevalence of $\mathrm{H}$. pylori infection among Myanmar children is common, as it is in other developing countries, and also highlight its association with socioeconomic status. Over time, the prevalence of $H$. pylori infections in children is expected to decline worldwide in parallel with the improvement in socioeconomic status of the nations. There is a need to intensify efforts of improving sanitation and living conditions in order to protect children against $H$. pylori infection.
\end{abstract}

Keywords: Helicobacter pylori, children, Myanmar, prevalence, seroprevalence

\section{INTRODUCTION}

Helicobacter pylori (H. pylori), first discovered by Warren and Marshall ${ }^{1,2}$ in 1982, a spiral-shaped pathogenic bacterium found on the human gastric mucosa, is prevalent worldwide. It has a strong association with chronic gastritis, peptic ulcer disease, gastric cancer and mucosa- associated lymphoid tissue lymphoma (MALT) ${ }^{3}$. The precise mechanisms of $H$. pylori transmission are not yet clear.

H. pylori is known to infect $50 \%$ of the world's population. An estimated $50 \%$ of children aged 5 years in developing countries are infected with $H$. pylori. It is thought that $H$. pylori infections are primarily acquired in childhood, with the incidence increasing gradually with age after the first few years of life $e^{4}$.

The prevalence of $H$. pylori is higher in low socioeconomic groups and in poor countries. Although the prevalence of $H$. pylori infection is declining in both developed and developing countries, it is still high in developing nations ${ }^{5}$ with prevalence of $60-80 \%{ }^{6}$. The overall prevalence of infection has been determined by epidemiological factors such as socioeconomic background, family composition, and availability of safe drinking water, basic hygiene and proper sanitation. Acquisition of $H$. pylori infection has been declining in developed countries at a faster rate than in developing countries, due to rapid improvement in hygienic practices and socioeconomic status of the countries ${ }^{7,8}$.

There are very few studies in Myanmar on $H$. pylori infection in children. The aim of this study was to determine the seroprevalence and associated factors of $H$. pylori infection among Myanmar children in Insein Township, which is a peri-urban community situated at the northern region of Yangon with a total population of 300,000 , of which, one-third are children under 16 years of age. Insein Township is located in the northern Yangon and is about 20 miles from the downtown of Yangon. The township comprises 21 wards, and shares borders with Shwepyitha Township in the north, Hlaingthaya Township in the west, and Mingalardon Township in the east and south. It is a slowly growing community, with the people mainly from poor to moderate social classes. 


\section{METHODS}

A community based cross sectional study was conducted in Insein Township in 2006.

A total of 193 children, aged between 2 and 13 years of both sexes, were recruited randomly from a list from 21 wards in the Insein Township. The validated questionnaire was used and the data were collected by the trained interviewers. Demographic characteristics such as age, sex, residence, level of education and occupation of parents, daily family income, family possessions, the number of family members, type of water supply, treatment of drinking water, type of latrine and type of house, were collected. Socioeconomic status was classified according to the modified scoring adapted from socioeconomic status scale by Kuppuswamy and Udai Pareek ${ }^{9}$.

\section{Collection and testing of the sample}

Two milliliters ( $\mathrm{mls}$ ) of blood was collected under aseptic measures and transferred to the laboratory on the day of collection for processing and stored at $2-8 \cdot C$. An in-house enzyme linked immunosorbent assay (ELISA), developed by Miyoshi et al., was used to determine serum $H$. pylori IgG antibodies. Both the sensitivity and specificity of the test was $90 \%$. The test was done in the Experimental Medicine Unit of the Department of Medical Research, Lower Myanmar.

\section{Data analysis}

The analysis of data involves different tabulation and cross correlation. Categorical variables were analyzed using Chi-square test and continuous variables were analyzed using two-sample t-test, comparing the $H$. pylori positive and negative groups. Statistical analysis was performed by utilizing the SPSS version 18 (IBM, New York, USA). $\mathrm{p}$-value $<0.05$ was considered statistically significant.

\section{Ethical consent}

Approval of this study was received from the Master Degree Protocol Board, University of Medicine 2, Myanmar and the informed consent for the study was obtained from the caregivers of the children involved.

\section{RESULTS}

A total of 193 children, with age ranging from 2 to 13 years were studied. The mean age of the participants was $7.6+/-3.85$ years. Out of 193 children, 130 children $(67.36 \%)$ were positive for $H$. pylori antibodies.

H. pylori antibody positivity was highest (84.37\%) among 8 - 11.9 years age group and lowest $(54.72 \%)$ among the $2-4.9$ years age group. The older the age, the more prevalent was the antibody positivity $(\mathrm{p}=0.006)$. The mean age of $H$. pylori positive children was $8.18+/-3.8$ and that of $H$. pylori negative children was $6.41+/-3.7$ $(p=<0.05)$. Those children positive for $H$. pylori infection were significantly older than those negative for $H$. pylori infection. Seroprevalence rates with reference to age and distribution are shown in Table 1, Figure 1 and 2.

There were 92 males and 101 females in the studied group and it was found that 64 out of 92 males $(69.57 \%)$ and 66 out of 101 females (65.35\%) were $H$. pylori antibody positive. However, no significant difference was seen between male and female $(p=0.532)$. The findings indicate that $H$. pylori infection can occur in both sexes without any sex predilection.

The subjects positive for $H$. pylori infection had a mean crowding index of $3.084+/-0.984$ and the negative ones had a mean crowding index of $2.92+/-1.04$. Thus, there was no significant association between $H$. pylori infection and overcrowding $(p-$ value $=0.309)$.

The children who tested positive for $H$. pylori infection had a mean family member of $5.97+/$ 2.22 and those who tested negative had $6.06+/$ 2.17. Thus, there was no association between $H$. pylori infection and number of family members $\mathrm{H}$. pylori positivity was detected in $14(63.64 \%), 18$ (69.23\%), $69(69.7 \%)$ and $29(63.04 \%)$ of those living in brick-houses, semi-brick houses, wooden houses and bamboo houses, respectively. There was no significant difference in the prevalence of $H$. pylori positivity and type of house.

Among 193 children, 19 children had drunk boiled water, 98 children filtered water, and 76 children untreated water. Positive $\mathrm{H}$. pylori antibodies were found in 11 (57.89\%), 71 (72.45\%) and 48 $(63.16 \%)$ of those using boiled, filtered and untreated drinking water, respectively. There was no significant association between $H$. pylori infection and type of drinking water (p value $=0.368$ ).

H. pylori antibodies were found in $63(64.29 \%), 58$ (71.6\%) and 9 (64.29\%) of those using pit latrines, flush latrines and surface latrines, respectively. Although it was found that $H$. pylori antibody positivity was highest amongst those using flush latrines, it was not statistically significant $(p=$ $0.535)$. H. pylori antibodies were positive in 102 (69.39\%), 21 (63.64\%) and 7 (53.85\%) children in low, middle and high socio-economic groups, respectively (Figure 3). Although it was not statistically significant, the prevalence rate was significantly lower $(53.85 \%)$ in children with high socio-economic status. Table 2 shows $H$. pylori seropositivity with reference to type of house, treatment of drinking water, type of latrine and socioeconomic status. 
Table 1: Prevalence of $H$. pylori antibody positive children according to age

\begin{tabular}{lcccc}
\hline Age (years) & \multicolumn{2}{c}{ H. pylori antibody } & Total & Positive rate \\
\hline & Positive & Negative & & \\
$2-4.9$ & 29 & 28 & 57 & $54.72 \%$ \\
$5-7.9$ & 42 & 19 & 61 & $68.85 \%$ \\
$8-10.9$ & 27 & 5 & 32 & $84.37 \%$ \\
$11-13.9$ & 32 & 11 & 43 & $74.42 \%$ \\
Total & 130 & 63 & 193 & $67.36 \%$ \\
\hline
\end{tabular}

Table 2: Type of house, type of drinking water, type of latrine and socioeconomic status in relation to $H$. pylori positivity rate

\begin{tabular}{lllll}
\hline Characteristics & $\begin{array}{c}\text { H. pylori } \\
\text { positive(n) }\end{array}$ & $\begin{array}{c}\text { H. pylori } \\
\text { negative(n) }\end{array}$ & $\begin{array}{c}\text { Positive rate } \\
(\%)\end{array}$ & p-value \\
\hline Type of house & & & 63.64 & 0.77 \\
Brick house & 14 & 8 & 69.23 & \\
Semi-brick house & 18 & 8 & 69.7 & \\
Wooden house & 69 & 30 & 63.04 & \\
Bamboo house & 29 & 17 & 57.89 & \\
& & & 72.45 & \\
Type of drinking water & 11 & 8 & 63.16 & \\
Boiled & 71 & 27 & & \\
Filtered & 48 & 28 & & \\
Untreated & & & & \\
& & & & \\
& & & 64.29 & \\
Type of latrine & 63 & 35 & 71.60 & \\
Pit & 58 & 23 & 64.29 & \\
Flush & 9 & 5 & & \\
Surface & & & 69.45 \\
Socio-economic status & 102 & 45 & 63.64 & \\
Low & 21 & 12 & 53.85 & \\
Middle & 7 & 6 & \\
High & & & \\
\hline
\end{tabular}

Figure 1: Distribution of $H$. Pylori infection according to age

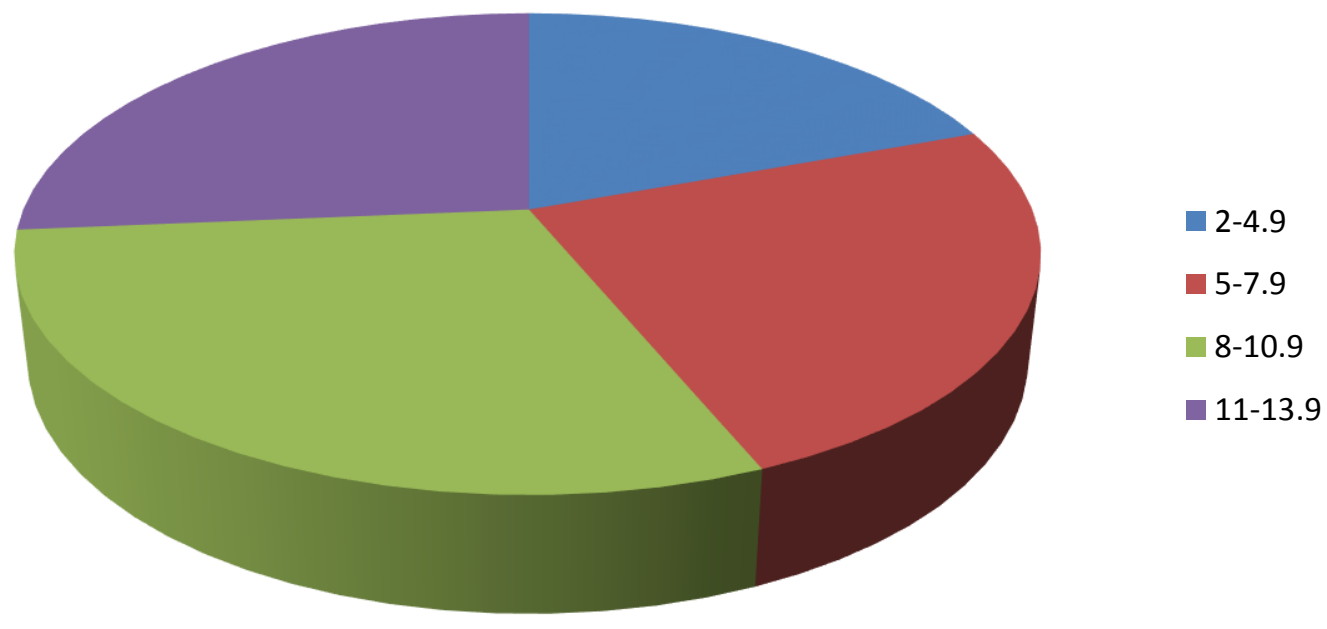


Figure 2: Prevalence of $H$. pylori infection by age group

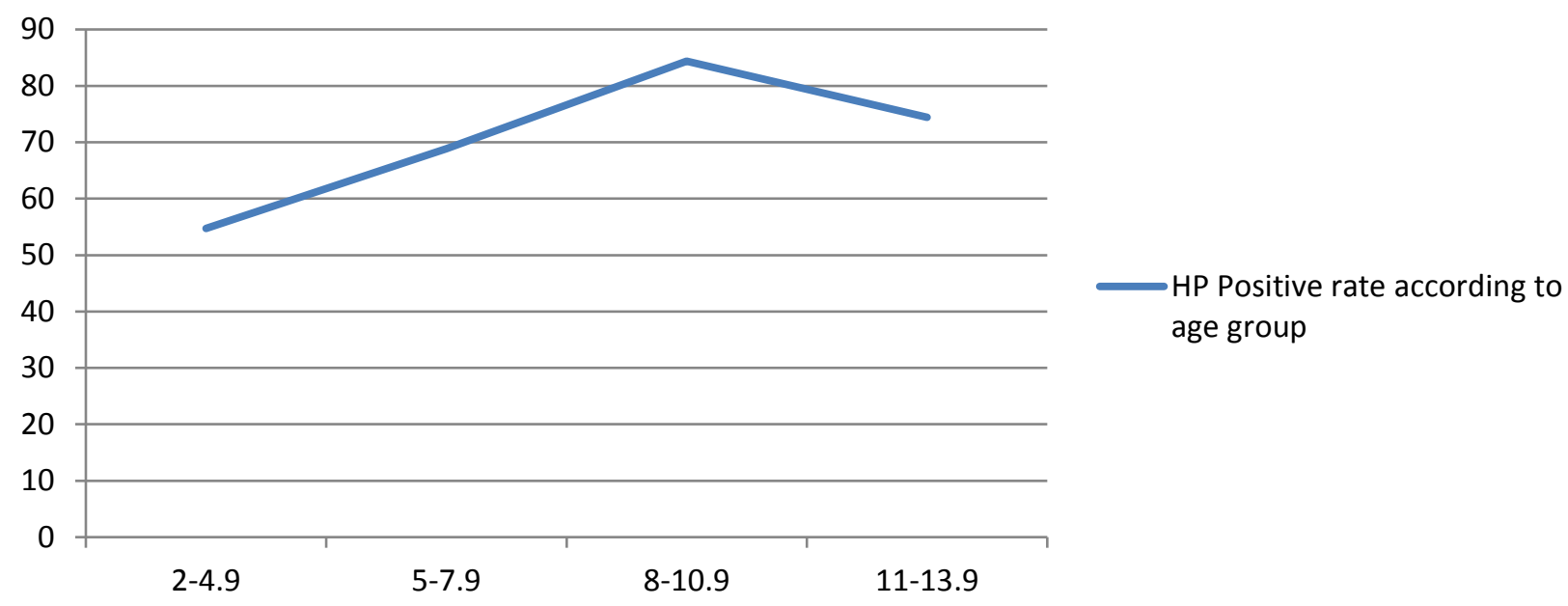

Figure 3: Prevalence of $H$. pylori infection in different socio-economic status

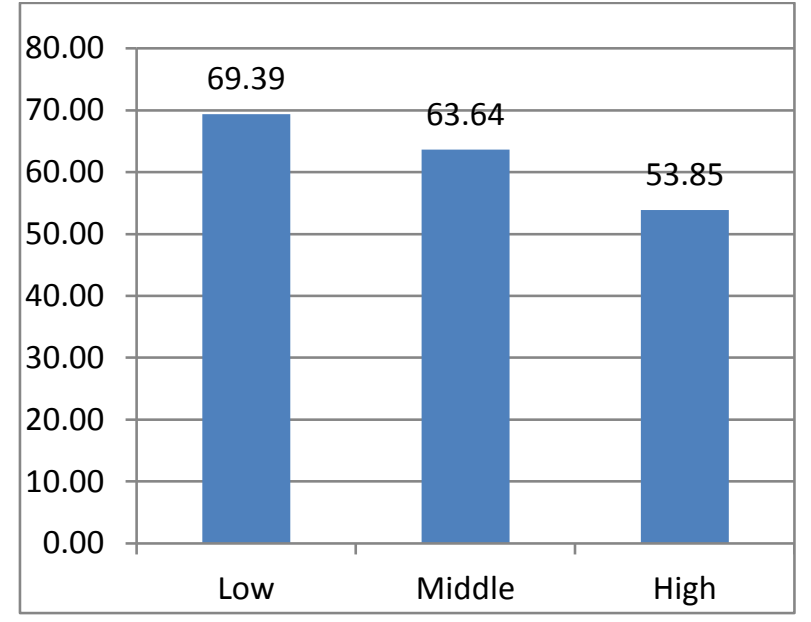

\section{DISCUSSION}

The prevalence of $H$. pylori infection in this study population was $67.36 \%$. Our finding is similar to those from other developing countries. The prevalence of $H$. pylori in children from Nigeria, aged 5 to 9 years, was $82 \%{ }^{10}$. In Bangladesh, the prevalence of $H$. pylori was $58 \%$ and $82 \%$ in children aged 0 to 4 years and 8 to 9 years, respectively ${ }^{10}$. In Sri Lanka, the prevalence was $67 \%$ in participants aged 6 to 19 years ${ }^{10}$. Santos et.al conducted a population based cross sectional study in Southern Brazil and reported the $H$. pylori infection prevalence rate of $63.4 \%{ }^{11}$, which is also consistent with the findings of present study. In a group of Myanmar children aged between 5 and 14 years attending a monastery school, which was an institution for children from rural areas, it was found that $70 \%$ of them were infected with $H$. pylori ${ }^{12}$.

Surprisingly, the prevalence of $H$. pylori in children from Vietnam, Taiwan and Korea was very low: $22.6 \%, 8.1 \%$ and $22 \%$, respectively $13,14,15$. Although these are developing countries, the mean income per inhabitant is much higher, which might explain the difference in $H$. pylori prevalence from those of other developing countries.

The prevalence of $H$. pylori in children had been much lower in developed countries: $8.6 \%, 2.4 \%$ and $3.7 \%$ in Ireland ${ }^{13}$ Germany $^{16}$, and Japan ${ }^{17}$, respectively. In Belgium, the prevalence of $H$. pylori infections in children and young adults was reported as $11 \%$. Among positive subjects, $3.2 \%$ were Belgian born children and $60 \%$ were born of immigrants from countries with a high prevalence of $H$. pylori infection ${ }^{18}$. A study done in St. Petersburg, Russia on children aged 2 to 19 years reported that $H$. pylori prevalence rates had declined from $44 \%$ in 1995 to $13 \%$ in 2005, which was probably related to the improvement in the standard of living over the last decade ${ }^{19}$.

In this study, $H$. pylori antibodies were most prevalent $(84.37 \%)$ among the $8-10.9$ years age group. The mean age of $H$. pylori-positive children was $8.18+/-3.8$ years and that of $H$. pylorinegative children was $6.41+/-3.7$ years. Those positive for $H$. pylori infection were significantly older than those who were negative. The infection rate was quite low $(54.72 \%)$ in children aged 2 to 4.9 years and rose steadily to reach its highest level between the ages of 8 to 10.9 years $(84.37 \%)$ and slightly fell to $74.42 \%$ between 11 and 13.9 years. The exact mechanism for this phenomenon has not yet been identified. There were reports of the decreasing prevalence of $H$. pylori infection over a brief interval in childhood ${ }^{20-23}$. One explanation for this phenomenon is spontaneous elimination of $H$. pylori possibly due to the natural history of the infection; and the other proposed concept is elimination of $H$. pylori through the use of antibiotics for other infections in older children $^{20,24-30}$.

The finding in this study is also consistent with that of Mégraud et. $a l^{6}$ and Sack and $\mathrm{Gyr}^{8}$ as they reported that by 5 years of age, about $50 \%$ of children in developing countries were already 
infected with $H$. pylori. In this study, there was no gender preponderance for $H$. pylori infection. Similar findings had been published in the study done in Texas $^{31}$, Southern China ${ }^{4}$, Peru ${ }^{32}$ and Taiwan $^{33}$.In our study, $H$. pylori infection was not associated with overcrowding in the family and there was no difference in distribution depending on the number of family members. However, in a population based cross sectional study done on children from West Iran, it was found that $H$. pylori positivity was significantly higher (71\%) among children with large family size; when compared to those with small family size $(53.2 \%)^{34}$. Moreover, in the study on Brazilian children, there was positive association between $H$. pylori infection and number of siblings and nursery attendance, which were considered as indicators of crowding ${ }^{35}$. Nursery attendance was not included in the variables in our study, which probably explains why the finding in the present study is different from others.

In this study, there was no association between $H$. pylori infection and type of building, type of drinking water and type of latrine, which is different from other studies. Generally, the likely modes of transmission are by direct contact, the oral-oral route (through vomitus and saliva) or the fecal-oral route. Goodman et al. ${ }^{36}$ also reported that children who swam in rivers or pools and used streams as a source of drinking water and those who frequently consumed raw vegetables were more likely to acquire $H$. pylori infection. In some parts of the world in which untreated water is used, waterborne transmission is considered as a major source of infection, probably due to fecal contamination ${ }^{11}$. In a study in Southern China, it was found that both place of residence and source of drinking water were significantly associated with $H$. pylori infection ${ }^{4}$. In this study, surprisingly, it was found that $H$. pylori positivity rate was higher in those using filtered water than those using untreated water. In this case, we need to consider on the source, in addition to the type of drinking water. Unfortunately, our data did not include the source of drinking water. In Insein Township, some households use municipal water and some use community wells. Klein et al. ${ }^{37}$ reported that households who got municipal water supply were 12 times more prone to be infected than those who got water from community well. So, we shall give a comment that the water source of the $H$. pylori positive children who consumed filtered water might be municipal water, for which further study is suggested.

In this study, although it is not statistically significant, the prevalence rate was lower (53.85\%) in children from families with high socioeconomic status, compared to those from families with low socio-economic status (69.39\%). In a study done in Italy, it was found that the prevalence of $H$. pylori infection in children was higher if the social conditions were lower $^{1}$. It has been reported that $H$. pylori infection rates were significantly higher in children of farmers compared to children of blue and white-collar families ${ }^{1}$. A study done among adults in southern Brazil also reported the association between low socioeconomic status and high $H$. pylori prevalence. These findings support the report of Malaty and Graham that a strong inverse correlation exists between childhood social class and $H$. pylori infection ${ }^{38}$. Overall, inadequate sanitation practices, low socioeconomic status and overcrowding or high-density living conditions seem to be related to a higher prevalence of $H$. pylori infection ${ }^{11}$.

The limitation of this study is that variables pertaining to feeding practices, such as duration of breast feeding, habit of sharing utensils, habit of eating raw vegetables, etc. were not included in the assessment. Regarding the household sanitation practice related to water used, our assessment was based on treatment of drinking water only. Instead, the clean water index (CWI) should have been implemented and source of drinking water should have been evaluated to get more accurate data.

\section{CONCLUSION}

Our findings suggest that the prevalence of $H$. pylori infection among Myanmar children is common, as it is in other developing countries, and also highlight its association with socioeconomic status. Over time, the prevalence of $H$. pylori infections in children is expected to decline worldwide in parallel with the improvement in socioeconomic status of the nations. There is a need to intensify efforts of improving sanitation and living conditions in order to protect children against $H$. pylori infection.

\section{Acknowledgement}

We would like to thank Dr. U Myo Khin, Retired Director General, Department of Medical Research, Myanmar for his close supervision and continuous support for this study. We also convey our sincere gratitude to Professor Dr. Zulkifli Bin Ahmad, Faculty of Medicine and Health Sciences, UCSI University for valuable advice on preparing this manuscript. We would also like to thank Dr. Yi Yi Kyaw, Research Scientist, Experimental Medicine Research Division, Department of Medical Research, Myanmar for her great help in collection, sampling and testing. Last but not least, we offer our gratitude to the children and their parents for their kind cooperation and willingness to participate in our study.

\section{REFERENCES}

1. World Health Organization. Breast 1. Warren JR, Marshall BJ. Unidentified curved bacilli on gastric epithelium in active chronic gastritis. Lancet 1983; 321:1273-1275. 
2. Marshall BJ, Warren JR. Unidentified curved bacilli in the stomach of patients with gastritis and peptic ulceration. Lancet 1984; 1:1311-1315.

3. Parsonnet J. The incidence of Helicobacter pylori infection. Aliment Pharmacol Therapeut 1995;9(suppl2):4551.

4. Mitchell HM, Li YY, Hu PJ, et al. Epidemiology of Helicobacter pylori in southern China: identification of early childhood as the critical period for acquisition. J Infect Dis 1992;166:149 153.

5. Torres J, Pérez-Pérez G, Goodman KJ, et al. A comprehensive review of the natural history of Helicobacter pylori infection in children. Arch Med Res 2000; 31: 431-469.

6. Mégraud F, Brassens-Rabbe MP, Denis F, et al. Seroepidemiology of Campylobacter pylori infection in various populations. $J$ Clin Microbiol 1989; 27:1870-1873.

7. The EUROGAST Study Group. Epidemiology of, and risk factors for, Helicobacter pylori infection among 3194 asymptomatic subjects in 17 populations. Gut 1993; 34:1672-1676.

8. Brown LM. Helicobacter pylori: epidemiology and routes of transmission. Epidemiol Rev 2000; 22:283-297.

9. Guru Raj M.S., Shilpa S., Maheshwaran, R. Revised socio-economic status scale for urban and rural India - Revision for 2015. The Scientific Journal for Theory and Practice of Socio-economic Development, 2015; 4(7): 167-174.

10. Organization WG. World Gastroenterology Organization Global Guideline: Helicobacter pylori in developing countries. J Clin Gastroenterol 2011; 45: 383-388.

11. Santos IS, Boccio J, Santos AS, Valle NC, Halal CS, Bachilli MC, Lopes RD: Prevalence of Helicobacter pylori infection and associated factors among adults in Southern Brazil: a populationbased cross-sectional study. BMC Public Health 2005; 5:118.

12. Flynn, Peter. The epidemiology of Helicobacter pylori in Myanmar [B.Sc(Med)Hons(Microbiology)Thesis]Sydn ey: University of New South Wales, 2000.

13. Rowland M, Kumar D, Daly L, O'Connor P, Vaughan D, Drumm B. Low rates of
Helicobacter pylori reinfection in children. Gastroenterol 1999;117:336-41.

14. Lin DB, Nieh WT, Wang HM, et al. Seroepidemiology of Helicobacter pylori infection among preschool children in Taiwan. Am J Trop Med Hyg 1999;61: 554558.

15. Malaty HM, Kim JG, Kim SD, Graham DY. Prevalence of Helicobacter pylori infection in Korean children: inverse relation to socioeconomic status despite a uniformly high prevalence in adults. Am J Epidemiol 1996;143: 257-262.

16. Weyermann $M$, Adler $G$, Brenner $H$, Rothenbacher $D$. The mother as source of Helicobacter pylori infection. Epidemiol 2006; 17:332-4.

17. Okuda M, Miyashiro E, Booka M, Tsuji T, Nakazawa T. Helicobacter pylori Colonization in the First 3 Years of Life in Japanese Children. Helicobacter 2007;12:324-7.

18. Mana F, Vandebosch S, Miendje Deyi V, et al. Prevalence of and risk factors for Helicobacter pylori in healthy children and young adults in Belgium anno 2010/2011. Acta Gastroenterol Belg 2013;76: 381-5.

19. Tkachenko MA, Zhannat NZ, Erman LV, Blashenkova EL, Isachenko SV, Isachenko $\mathrm{OB}$, et al. Dramatic changes in the prevalence of Helicobacter pylori infection during childhood: a 10-year follow-up study in Russia. $J$ Pediatr Gastroenterol Nutr.2007;45:428-32.

20. Redlinger T, O'Rourke K, Goodman KJ. Age distribution of Helicobacter pylori seroprevalence among young children in a United States/Mexico border community: evidence for transitory infection. $A m \mathrm{~J}$ Epidemiol 1999;150(3):225-30.

21. Lindkvist $P$, Asrat D, Nilsson I, et al. Age at acquisition of Helicobacter pylori infection: comparison of a high and a low prevalence country. Scand J Infec Dis 1996;28(2):181-4.

22. Blecker U, Hauser B, Lanciers S, et al. The prevalence of Helicobacter pylori-positive serology in asymptomatic children. $J$ Pediatr Gastroenterol Nutr 1993;16(3):252.

23. Mahalanabis D, Rahman MM, Sarker SA, et al. Helicobacter pylori infection in the young in Bangladesh: prevalence, socioeconomic and nutritional aspects. Int J Epidemiol 1996;25 (4):894-8. 
24. Rothenbacher D, Bode $G$, Brenner $H$. Dynamics of Helicobacter pylori infection in early childhood in a high-risk group living in Germany: loss of infection higher than acquisition. Aliment Pharmacol Ther 2002;16(9):1663-8.

25. Perri F, Pastore $\mathrm{M}$, Clemente $\mathrm{R}$, et al. Helicobacter pylori infection may undergo spontaneous eradication in children: a 2 year follow-up study. J Pediatr Gastroenterol Nutr 1998;27(2):181-3.

26. Xia H, Talley N. Natural acquisition and spontaneous elimination of Helicobacter pylori infection: clinical implications. Am J Gastroenterol 1997;92(10):1780-7.

27. Hestvik E, Tylleskar T, Kaddu-Mulindwa D, et al. Helicobacter pylori in apparently healthy children aged 0-12 years in urban Kampala, Uganda: a community-based cross sectional survey. BMC Gastroenterol 2010;10:62.

28. Malaty HM, El-Kasabany A, Graham DY, et al. Age at acquisition of Helicobacter pylori infection: a follow-up study from infancy to adulthood. Lancet 2002;359(9310):931-5.

29. Malaty HM, Graham DY, Wattigney WA, et al. Natural history of Helicobacter pylori infection in childhood:12-year follow-up cohort study in a biracial community. Clin Infect Dis 1999;28(2):279-82.

30. Broussard CS, Goodman KJ, Phillips CV, et al. Antibiotics taken for other illnesses and spontaneous clearance of Helicobacter pylori infection in children. Pharmacoepidemiol Drug Saf 2009;18(8):722-9.

31. Fiedorek SC, Malaty HM, Evans DL, Factors influencing the epidemiology of Helicobacter pylori infection in children. Pediatrics 1991; 88:578-582

32. Klein P D, Graham D Y, Gaillover A, Opekun A R, Smith E O, Water source as risk factor for Helicobacter pylori infection in Peruvian children. The Lancet 1991;337:1503-05

33. The $B \mathrm{H}$, Lin $\mathrm{J} \mathrm{T}$, Pan $\mathrm{WH}$, Lin $\mathrm{S} \mathrm{H}$, Wang L Y, Lee T K, Chen C J. Seroprevalence and associated risk factors of Helicobacter pylori infection in Taiwan. Anticancer-Res $1994 \quad$ MayJune; 14(3B):1389-92

34. Jafar S, Jalil A, Soheila N, Sirous S. Prevalence of Helicobacter pylori infection in children, a population-based cross-sectional study in west Iran. Iran J Pediatr. 2013; 23: 13-8.

35. Dattoli V. C., Veiga R. V., da Cunha S. S., Pontes-de-Carvalho L. C., Barreto M. L., Alcântara-Neves N. M. Seroprevalence and potential risk factors for Helicobacter pylori infection in Brazilian children. Helicobacter 2010;15(4);273-8.

36. Goodman KJ, Correa P, Tengana Aux HJ, et al. Helicobacter pylori infection in the Colombian Andes: a population-based study of transmission pathways. Am J Epidemiol. 1996;144:290-9.

37. Klein PD, Graham DY, Gaillour A, et al. Water source as risk factor for Helicobacter pylori infection in Peruvian children. Gastrointestinal Physiology Working Group. Lancet 1991;337:1503-6.

38. Malaty HM, Graham DY: Importance of childhood socioeconomic status on the current prevalence of Helicobacter pylori infection. Gut 1994; 35:742-745. 